\title{
USE OF POLARIZATION SPECTROSCOPY IN STUDIES OF LANTHANIDE COMPLEXES IN SOLUTION
}

\author{
J.P. RIEHL \\ Department of Chemistry, Michigan Technological University \\ Houghton, MI 49931, USA
}

\begin{abstract}
The use of linearly or circularly polarized light in the absorption or excitation beam in spectroscopic studies involving lanthanide ions, and the analysis of emitted light polarization has provided useful information concerning molecular structure, and excited state dynamics and energetics. Linear polarization studies may be used to aid in the assignment of crystal field components of electronic transitions, and circular polarization may be employed as a probe of chiral structure and structural changes. Examples of several of the different experimental techniques are presented and discussed.
\end{abstract}

PACS numbers: 78.60.-b

\section{Introduction}

Luminescence spectroscopy involving the intraconfigurational $f \leftrightarrow f$ transitions of lanthanide ions has been widely used as a structural probe in biomolecular systems $[1,2]$, and in studies concerned witl the structure and speciation of lanthanide complexes in solution, and in the solid state [3]. In recent years the use of linearly or circularly polarized light in the absorption or excitation beam in spectroscopic studies involving lanthanide ions, and the analysis of emitted light polarization has also provided useful information concerning molecular structure, and excited state dynamics and energetics [4-7].

For purpose of discussion it is useful to separate spectroscopic experiments involving light polarization into three categories. In the first type of experiment the polarization of an incident excitation beam is modulated between either $x$ or $y$ linear polarization (assuming that the direction of propagation of the beam defines the laboratory $z$ axis), or between left or right circular polarization, and the intensity of the transmitted, or emitted beam is monitored. In the second type of experiment, the state of linear or circular polarization of the transmitted, or emitted beam is analyzed, and in the third type of experiment the incident beam polarization is controlled (or modulated) and the polarization of the exiting beam is simultaneously analyzed for its state of polarization. 
The effect of linear polarization in an excitation or absorption beam is to "photoselect" molecules based upon the orientation of the absorption dipole moment with the direction of linear polarization [8]. The linear polarization of an emitted beam reflects the orientational distribution of excited states. This is in contrast to studies involving circular polarization in which differences in absorption, or emission are due to intrinsic "chiral" molecular structure. Chiral or "optically active" molecules are those possessing a symmetry in which the mirror image isomers are non-superimposable. Almost all biologically important molecules are, in fact, chiral, therefore circular dichroism (CD) and circularly polarized luminescence (CPL) experiments have found widespread application in biochemistry. It should be mentioned that it is sometimes necessary to consider both the effects of linear and circular polarization as discussed below.

In this paper we will be concerned only with the use of polarization spectroscopic methods in studies of lanthanide ions in solutions, and as a result we will not discuss linear dichroism (LD) experiments, since for the reasons given above, this technique is only suitable for studying oriented samples. We will also not consider magnetic-field induced polarization spectroscopy, although this is a useful technique for studying lanthanide ions in absorption and emission $[9,10]$.

\section{Incident beam polarization modulation}

Since the extinction coefficients of most lanthanide (III) transitions are very small (usually $<1$ ), it is not surprising that CD techniques have not found extensive use as a probe of ground state chiral structure. In CD one usually reports the ratio of the differential absorbance $\Delta A$ to the total absorbance $A$. This defines the so-called absorption dissymmetry factor, $g_{\mathrm{abs}}(\lambda)$ which is related to the extinction coefficients for left $(\mathrm{L})$ and right $(\mathrm{R})$ circularly polarized light as follows:

$$
g_{\mathrm{abs}}(\lambda)=\frac{\Delta A(\lambda)}{A(\lambda)}=\frac{\Delta \varepsilon(\lambda)}{\varepsilon(\lambda)}=\frac{\varepsilon_{\mathrm{L}}-\varepsilon_{\mathrm{R}}}{\frac{1}{2}\left(\varepsilon_{\mathrm{L}}+\varepsilon_{\mathrm{R}}\right)} .
$$

As opposed to most organic cliromophores, $f \leftrightarrow f$ transitions which obey magnetic dipole selection rules, i.e. $\Delta J=0, \pm 1$ may have large dissymmetry factors [11]. However, measurement of differences in absorptivities $(\Delta A)$ of less than $10^{-4}$ is quite difficult. Because the concentration of most biomolecular samples is usually less than $10^{-3} \mathrm{M}$, and higher concentrations of lanthanide complexes often lead to oligomers or precipitation, one is practically limited to fairly dilute solutions in long path length cells. There have been few reported measurements of this type, however, to date no specific structural information has been derived from these difficult measurements [12].

\section{Polarization analysis of luminescence}

The analysis of the linear polarization of luminescence from molecules in solution obtained at $90^{\circ}$ from the excitation direction is routinely used to determine the degree of reorientation which has taken place between the absorption and emission. This information can then be used in concert with simple models to estimate molecular size [13]. These experiments are only possible if the lifetime of the excited state is such that the photoselected distribution prepared by the 
initial excitation beam has not been completely randomized by the time of emission. In the case of the luminescent lanthanide complexes of $\mathrm{Tb}(\mathrm{III})$ and $\mathrm{Eu}(\mathrm{III})$, the lifetimes are so long (usually $\mathrm{ms}$ ) that essentially complete randomization of the photoselected distribution occurs and, as a result, no linear polarization is observed under ordinary solution conditions. Although this property of lanthanide complexes does limit the usefulness of linear polarization measurements, the fact that there is no linear polarization is an important factor in the measurement of circular polarization due to imperfections in the optical devices used for these measurements [14].

Linearly polarized measurements may be useful as a probe of the symmetry of the $f \leftrightarrow f$ transitions for very viscous samples, e.g. glasses, in which the molecules can be assumed to be "frozen". Meskers et al. have shown, for example, how the measurement of the linear polarization (or depolarization) of crystal field transitions of the $D_{3}$ complex $\mathrm{Eu}(2,6 \text {-pyridine-dicarboxylate }=\mathrm{DPA})_{3}^{3-}$ may be used to assign the individual crystal field components [15]. This type of study is possible because of the low degeneracy of the Eu(III) states involved in the absorption and luminescence transitions, and the fact that in some cases the individual crystal field transitions can be resolved.

The measurement of the usually small net circular polarization in the luminescence (CPL) from chiral lanthanide complexes, and lanthanide ions as substitutional replacements of $\mathrm{Ca}$ (II) and $\mathrm{Fe}$ (III) in metal binding proteins has proven to be a useful probe of chiral molecular and biomolecular structure $[6,16]$. In CPL spectroscopy one measures the luminescence dissymmetry factor, $g_{\text {lum }}$, which is related to the intensity of left and right circularly polarized emitted light as follows [5]:

$$
g_{\operatorname{lum}}(\lambda)=\frac{\Delta I(\lambda)}{I(\lambda)}=\frac{I_{\mathrm{L}}-I_{\mathrm{R}}}{\frac{1}{2}\left(I_{\mathrm{L}}+I_{\mathrm{R}}\right)} .
$$

As mentioned above, $f \leftrightarrow f^{\prime}$ transitions that obey magnetic dipole selection rules often have very large intrinsic $g_{\text {lum }}$ values [11]. In Fig. 1 we show an approximate energy level diagram for the two most luminescent lantlianide ions, Eu(III) and $\mathrm{Tb}(\mathrm{III})$. The most intense emissive transition of $\mathrm{Tb}(\mathrm{III}),{ }^{5} D_{4} \rightarrow{ }^{7} F_{5}$, satisfies the above selection rules, and it is by far the most widely studied transition in CPL involving lanthanide ions. In the case of $\mathrm{Eu}(\mathrm{III})$, the transition from ${ }^{5} D_{0} \rightarrow{ }^{7} F_{1}$, although much weaker than the hypersensitive ${ }^{5} D_{0} \rightarrow{ }^{7} F_{2}$ transition is expected to have the larger $g_{\text {lum }}$ value.

Unlike many transition metal systems, no chiral complexes of lanthanide (III) ions containing achiral ligands have been isolated in enantiomeric forms due to the fact that the complexes are labile. It is possible in some cases to perturb the racemic ground state equilib) ium through the addition of non-coordinating chiral environment compounds $[17,18]$. An example of such an experiment is presented in Fig. 2. In this figure we plot the total luminescence and circularly polarized luminescence from an aqueous solution of the $\mathrm{D}_{3}$ complex $\mathrm{Tb}(2,6 \text {-pyridinedicarboxylate })_{3}^{3-}$ in which $1.0 \mathrm{M}$ of the sugar fructose has been added. The confirmation that this is simply a perturbation of the racemic equilibria, and not the formation of some new complex is provided by a comparison with a photoenriched sample as described below. It should be noted that these types of effects have been shown to depend 


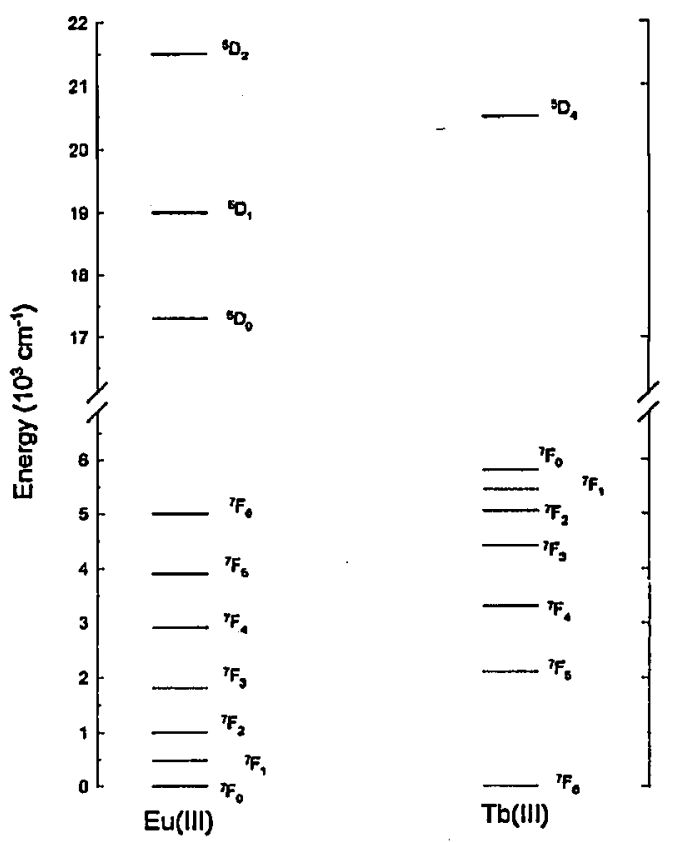

Fig. 1. Schematic approximate energy level diagram for $\mathrm{Eu}(\mathrm{III})$ and $\mathrm{Tb}(\mathrm{III})$.

linearly on the concentration of the chiral adduct, although to date no structural correlation between the chiral structure of the adduct and the direction of the equilibrium perturbation has yet been found [18].

It has also been demonstrated recently that non-racemic excited states can be generated from racemic ground states through enantioselective excited state quenching [19-23]. These experiments rely on the differential diastereomeric interaction between chiral acceptors and the racemic donor lanthanide complexes. The donors which have been used, have all been kinetically stable transition metal complexes. The quenching results have been analyzed in terms of kinetic models involving encounter pair complex formation, and the various thermodynamic activation parameters. CPL from complexes of $\mathrm{Tb}(\mathrm{III})$ and $\mathrm{Eu}$ (III) with chiralligands often yield very large $g_{\text {lum }}$ values, however, the usefulness of these measurements as specific structural probes awaits more progress in the development of reliable spectra-structure correlations [5].

The use of CPL from lanthanide ions in the study of chiral biological systems holds great promise as a selective site specific probe of chiral structure and structural changes [6]. As an example, in Fig. 3 we show the total emission and circularly polarized emission for a sample of transferrin in which $\mathrm{Tb}$ (III) has been bound to a site normally occupied by Fe(III) [24]. The spectral region shown corresponds to the ${ }^{5} D_{4} \rightarrow{ }^{7} F_{5}$ transition of $\mathrm{Tb}$ (III). As can be seen in this figure, the differential signals which can be obtained from lanthanides bound to these chiral systems can be quite large. In Fig. 4 , we show integrated $g_{\text {lum }}$ values for one of 

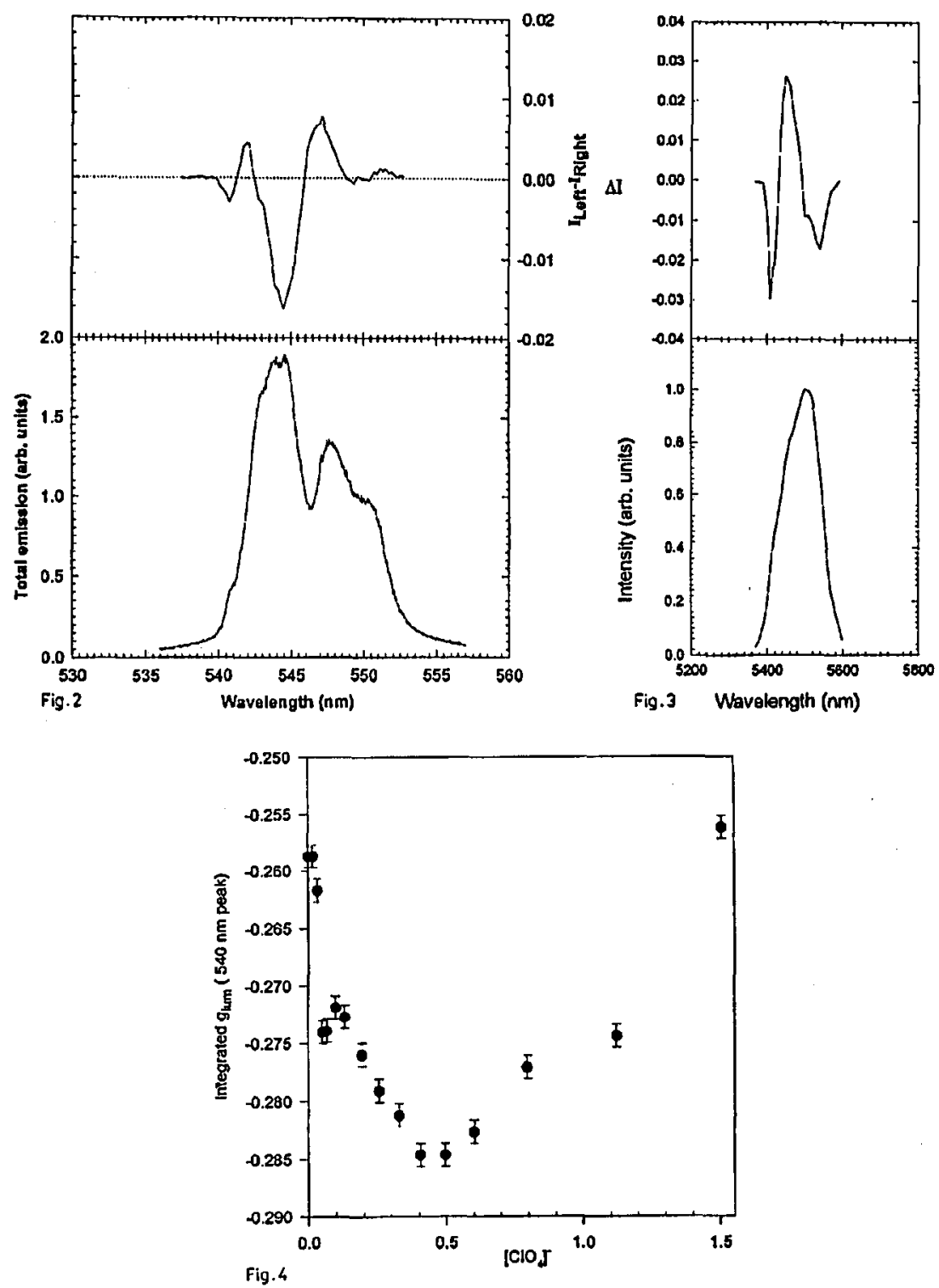

Fig. 2. Circularly polarized luminescence, $\Delta I$, and total luminescence, $I$, for a solution of $\mathrm{Tb}(2,6 \text {-pyridinedicarboxylate })_{3}^{3-}$ in which $1.0 \mathrm{M}$ fructose has been added. The spectral region shown corresponds to the ${ }^{5} D_{4} \rightarrow{ }^{7} F_{5}$ transition. $\lambda_{\text {exc }}=488 \mathrm{~nm}$.

Fig. 3. Circularly polarized luminescence, $\Delta I$, and total luminescence, $I$, for a solution of $\mathrm{Tb}(\mathrm{III})$ bound to serum transferrin. The spectral region shown corresponds to the ${ }^{5} D_{4} \rightarrow{ }^{7} F_{5}$ transition. $\lambda_{\text {exc }}=488 \mathrm{~nm}$.

Fig. 4. Effect of added perclilorate ion concentration on the integrated dissymmetry value for the peak at $541 \mathrm{~nm}$ for $\mathrm{Tb}$ (III) bound to serum transferrin. See Fig. 3. 
the peaks in the spectrum as a function of the concentration of added perchlorate ion. The total emission intensity decreases as the concentration of perchlorate ion increases, however, the effect of this ion on the chirality of the bound $\mathrm{Tb}$ (III) is such that initially the chirality increases, and then decreases. The effect of added anions on the structure of proteins such as transferrin is an area of general interest.

\section{Polarized excitation and polarized emission}

As mentioned above, the analysis of linear polarization in the luminescence spectrum of lanthanide complexes in isotropic rigid samples may be a useful technique to probe the symmetry of selected crystal field transitions. Since these experiments are always performed at right angles to the direction of excitation, the orientational distribution of rigid emitting species will not be isotropic even if the ground state is isotropic. It is more common however to use linearly polarized excitation, and then to report the polarization (or depolarization) of the emitting beam in terms of polarization directions parallel or perpendicular to the excitation polarization. This is, in fact, how the experiments described in Ref. [15] were performed. The use of linearly polarized excitation instead of unpolarized excitation has an additional advantage in that resultant polarization factors are larger.

The use of circularly polarized excitation in CPL studies of lanthanide complexes has found applications in a number of studies [25-29]. This is, in fact,

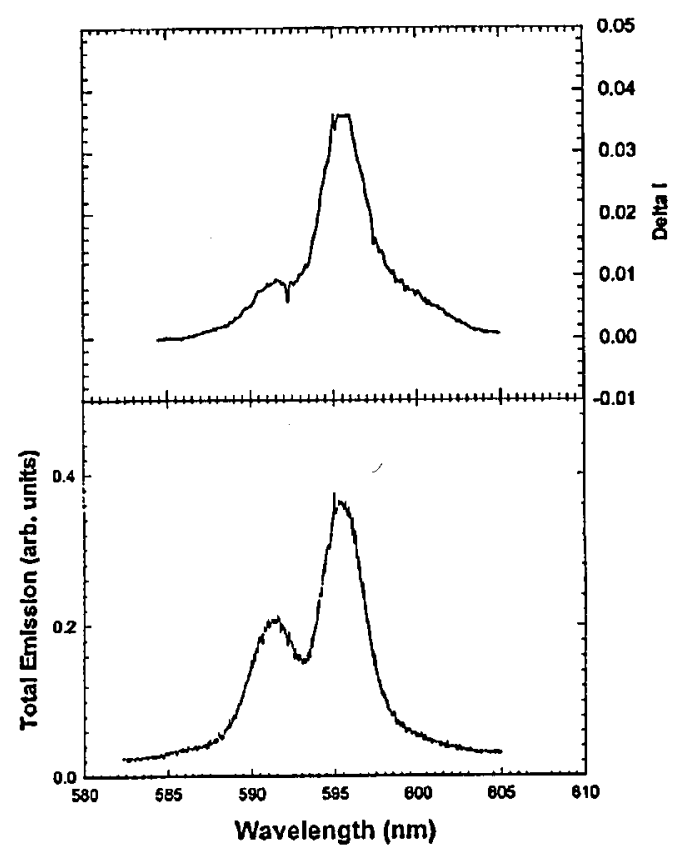

Fig. 5. Circularly polarized luminescence, $\Delta I$, and total luminescence, $I$, for an aqueous solution of $\mathrm{Eu}(\mathrm{DPA})_{3}^{3-}$. The spectral region shown corresponds to the ${ }^{5} D_{0} \rightarrow{ }^{7} F_{1}$ transition. $\lambda_{\text {exc }}=557.1 \mathrm{~nm}$. 
another way of preparing non-racemic excited states from racemic ground states. The only requirement here is that the rate of racemization must be slower than the emission lifetime. Ililmes and Riehl [30] have shown that the measured $g_{\text {lum }}$ value may be related to the intrinsic $g_{\text {lum }}$ and $g_{\mathrm{abs}}$ value of the pure enantiomers as follows:

$$
g_{\text {lum }}(\lambda)_{\text {measured }}=\frac{1}{2} g_{\mathrm{abs}}^{\mathrm{R}}\left(\lambda^{\prime}\right) g_{\text {lum }}^{\mathrm{R}}(\lambda) .
$$

$\lambda^{\prime}$ in this equation denotes the excitation wavelength, and the superscript $\mathrm{R}$ denotes one of the two possible enantiomers. Since for most systems the experimental limit on the measurement of glum is approximately $10^{-4}$, it follows that the enantiomeric $g_{\text {lum }}$ or $g_{\mathrm{abs}}$ values need to be quite large. This requirement limits the application of this experimental technique to transitions with relatively large dissymmetry values. As mentioned above, selected lanthanide transitions may possess very large dissymmetry values, and, as a result, this technique is particularly well suited for studying racemic lanthanide complexes.

In Fig. 5 we show CPL and total emission spectra for an aqueous solution of $\mathrm{Eu}(\mathrm{DPA})_{3}^{3-}$ following circularly polarized excitation at $557.1 \mathrm{~nm}$ from a CW dye laser. This excitation wavelength corresponds to the transition from the thermally excited ${ }^{7} F_{2}$ a probe of whether or not a complex is chiral, or as a probe of the rate of racemization of a complex known to be chiral.

\section{Acknowledgments}

Acknowledgment is made to the donors of the American Chemical Society - Petroleum Research Fund for partial support of this work. The author also acknowledge several helpful discussions and collaboration with Ewa IIuskowska, Kristen C. Smith, and Christine L. Maupin.

\section{References}

[1] R.B. Martin, F.S. Richardson, Quart. Rev. Biophys. 12, 181 (1979).

[2] W. De W. Horrocks Jr, D.R. Sudnick, Acc. Chem. Res. 14, 384 (1989).

[3] J. Reuben, in: Handlook on the Physics and Chemistry of Rare Earths, Vol. 4, Eds. K.A. Gschneidner, L. Eyring, North-Holland, Amsterdam 1979, p. 515.

[4] F.S. Richardson, J.P. Riehl, Chem. Rev. 77, 773 (1977).

[5] J.P. Riehl, F.S. Richardson, Chem. Rev. 86, 1 (1986).

[6] J.P. Riehl, F.S. Richardson, Meth. Enzymol. 22G, 539 (1993).

[7] J.P. Riehl, in: Analytical Applications of Circular Dichroism, Eds. N. Purdie, H.G. Brittain, Elsevier, Amsterdam 1993, Ch. 7, p. 207.

[8] A.C. Albrecht, J. Mol. Spectrosc. 6, 84 (1961).

[9] J.P. Riehl, F.S. Richardson, J. Chem. Phys. 68, 4266 (1978).

[10] J.P. Riehl, F.S. Richardson, J. Chem. Phys. 66, 1988 (1977).

[11] F.S. Richardson, Inorg. Chem. 19, 2906 (1980).

[12] L.I. Katzin, Coord. Chem. Rev. 5, 279 (1970).

[13] P.P. Feofilov, The Physical Basis of Polarized Emission, Consultants Bureau, New York 1961. 
[14] H.P.J.M. Dekkers, P. Moraal, J.M. Timper, J.P. Riehl, Appl. Spectrosc. 39, 3203 (1985).

[15] S.C.J. Meskers, H.P.J.M. Dekkers, J.P. Riehl, Chem. Phys. Lett. 216, 241 (1993).

[16] S. Abdollahi, W.R. Harris, J.P. Riehl, J. Phys. Chem. 100, 1950 (1996).

[17] S. Wu, G.L. Hilmes, J.P. Riehl, J. Plıys. Chem. 03, 2307 (1989).

[18] E. Huskowska, J.P. Riehl, Inorg. Chem. 34, 5615 (1995).

[19] D.P. Glover-Fischer, D.II. Metcalf, J.P. Bolender, F.S. Richardson, Chem. Phys. 198, 207 (1995).

[20] R.B. Rexwinkel, S.C.J. Meskers, J.P. Riehl, H.P.J.M. Dekkers, J. Phys. Chem. 96, 1112 (1992).

[21] R.B. Rexwinkel, S.C.J. Meskers, H.P.J.M. Dekkers, J.P. Riehl, J. Phys. Chem. 96, 5725 (1992).

[22] R.B. Rexwinkel, S.C.J. Meskers, J.P. Riehl, H.P.J.M. Dekkers, J. Phys. Chem. 07, 13519 (1993).

[23] R.B. Rexwinkel, S.C.J. Meskers, H.P.J.M. Dekkers, J.P. Riehl, J. Phys. Chem. 97, 3875 (1993).

[24] S. Abdulalli, W.R. Harris, J.P. Riehl, to be published.

[25] D.H. Metcalf, S.W. Snyder, J.N. Demas, F.S. Richardson, J. Am. Chem. Soc. 112, 5681 (1990).

[26] G.L. Ililmes, J.M. Timper, J.P. Riehl, Inorg. Chem. 24, 1721 (1985).

[27] G.L. Hilmes, N. Coruh, J.P. Riehl, Inorg. Chem. 27, 3647 (1988).

[28] G.L. Hilmes, N. Coruh, J.P. Riehl, Inorg. Chem. 27, 1136 (1988).

[29] A. Mondry, S.C.J. Meskers, J.P. Riehl, J. Lumin. 62, 17 (1994).

[30] G.L. Hilmes, J.P. Riehl, J. Phys. Chem. 87, 3300 (1983). 